\title{
Sertraline and curcumin prevent stress-induced morphological changes of dendrites and neurons in the medial prefrontal cortex of rats
}

\author{
Ali Noorafshan ${ }^{1,2}$, Mohammad-Amin Abdollahifar ${ }^{2}$, Saied Karbalay-Doust ${ }^{1,2}$, Reza Asadi-Golshan ${ }^{2}$, \\ Ali Rashidian-Rashidabadi² \\ ${ }^{1}$ Histomorphometry and Stereology Research Centre, Shiraz University of Medical Sciences, Shiraz, ${ }^{2}$ Anatomy Department, \\ School of Medicine, Shiraz University of Medical Sciences, Shiraz, Iran
}

\begin{abstract}
Stress induces structural and behavioral impairments. The changes in dendrites and neurons are accompanied by impairments in the tasks mediated by the medial prefrontal cortex (MPFC). The present study was conducted to evaluate the structural changes of the dendrites and neurons of the MPFC after stress using stereological methods. In addition, the effects of a natural and a synthetic substance, i.e., curcumin and sertraline, were evaluated. The rats were divided into 7 groups: stress + distilled water, stress + olive oil, curcumin (100 mg/kg/day), sertraline (10 mg/ $\mathrm{kg} /$ day), stress + curcumin, stress + sertraline, and control groups. The animals were submitted to chronic variable stress for 56 days. The results showed an average 15\% reduction in the length of the dendrites per neuron in the mPFC after stress $(p<0.004)$. The total spine density was reduced by $50 \%$ in the stress (+ olive oil or + distilled water) groups in comparison with the control group $(p<0.01)$. The main reduction was seen in the thin and mushroom spines, while the stubby spines remained unchanged. Mean volume and surface area of the neurons were decreased by $14 \%$ and $10 \%$ on average in the stress (+ distilled water or + olive oil) rats in comparison to the control rats, respectively $(p<0.01)$. The data revealed that treatment of stressed rats with curcumin or sertraline can prevent the loss of spines and reduction of dendrite length, volume and surface area of the neurons. Sertraline and curcumin can prevent structural changes of the neurons and dendrites induced by stress in the MPFC of rats.
\end{abstract}

Key words: stress, sertraline, curcumin, cortex, dendrite, neuron, stereology.

\section{Introduction}

Chronic stress is perceived and the response is coordinated by the brain. It has been well documented that uncontrollable stress induced dendritic remodeling in several brain regions $[37,39]$. It has been reported that chronic stress for 21 days reduced the length as well as the number of dendrites and spines in the hippocampus [5]. The chronic stressinduced morphological changes may be correlated with the specialized functions of the prefrontal cortex (PFC) sub-regions in stress-related pathologies $[30,31]$. Alterations in spine shape (stubby, mushroom or thin shaped) and number have been reported in 
neurodegenerative disorders, including depression $[2,28]$. Previous studies have shown morphological changes in the pyramidal neurons in the PFC following chronic stress [30,31]. Our previous study showed that stress affected behavioral tests, such as spatial learning and memory, anxiety, and anhedonia [23]. These behavioral changes might be accompanied by structural remodeling of the neurons including their dendrites. It has been reported that the changes in dendritic length and spine density are accompanied by impairments in the cognitive tasks selectively mediated by the mPFC [18]. These reports suggest that stress-induced remodeling in the mPFC may have distinct functional consequences. Previous studies have also demonstrated that chronic restraint stress changed the morphology of the neurons in the mPFC [32]. It has been reported that the prefrontal cortex shows alterations in the cerebral structures in depressed patients [19]. In addition, previous investigations have indicated reduced neuronal soma size in the prefrontal cortex [29,32]. In spite of these reports that reveal the possible chronic stress-induced morphological changes of neurons in the MPFC, no studies have been carried out on the neuron volume, volume of neuron nuclei and neuron surface area.

Medial PFC plays a key role in controlling the hypothalamic-pituitary-adrenal (HPA) axis and regulates the stress response of other structures [8]. Depression shows a good response to pharmacological treatments, and among the various drug agents, selective serotonin reuptake inhibitors (SSRIs) and tricyclic antidepressants (TCAs) are widely used in adult patients [25]. Sertraline is an antidepressant which is used to treat major depression in adult patients. Its antioxidant and neuroprotective actions have also been reported in neurodegenerative diseases [43]. Curcumin is the principal curcuminoid of the popular Indian spice turmeric. It has been used to manage stress and depression related disorders in China [35] and is known to have anti-inflammatory, antioxidant, anti-carcinogenic, anti-microbial, and neuroprotective effects $[3,16]$. The previous studies showed that antidepressant like action of curcumin, its use in major depression, these including, inhibitor of monoamine oxidase (MAO) enzyme, regulations the level of various neurotransmitters, promotes hippocampal [17]. In another study, curcumin was shown to protect the dopamine-producing cells of the substantial nigra area of the brain in a rat model of Parkinson's disease [17].
The present study aimed to evaluate and compare the effects of a natural and a synthetic substance, i.e., curcumin and sertraline, on stress in an animal model. Design-based stereological methods were used to estimate the total dendritic length per neuron, density and morphology of the spines, mean volume of the neuron and nucleus and mean surface area of the neuron in the MPFC of rats. Mean volume was estimated using the nucleator method. Estimation of the neuron surface area received less attention and here the surfactor method was used for the estimation. In the method of length estimation presented here, there is no need for the time-consuming method of dendrite tracing using instruments such as the camera lucida. In addition, in the tracing method, some dendrites that are anterior or posterior to the dendrite might not be identifiable by the researchers.

\section{Material and methods}

\section{Animals}

In this study, 42 adult male Sprague-Dawley rats (240 to $280 \mathrm{~g}$ ) were obtained from the Laboratory Animal Center of Shiraz University of Medical Sciences, Shiraz, Iran. The Ethics Committee of the University approved the animal experiment (Approval No. 91-6124). The male rats were randomly assigned to experimental and control groups. Each group included 6 rats that were housed under standard conditions, room temperature $\left(22-24^{\circ} \mathrm{C}\right)$, and a $12: 12 \mathrm{~h}$ light-dark schedule and had free access to water and food. The animals were divided into seven groups: (I) stress + water group daily receiving stress and distilled water, (II) stress + olive oil group daily receiving stress and olive oil, (III) curcumin group receiving curcumin (100 mg/kg/day) [23,24], (IV) sertraline group receiving sertraline (10 mg/kg/day) [43], (V) stress + curcumin group daily receiving stress and curcumin (100 mg/kg/day) [23,24], (VI) stress + sertraline group daily receiving stress and sertraline (10 mg/kg/day) [43], and (VII) the control group. All the animals received $1 \mathrm{ml}$ of the medications by gavage.

\section{Stress model}

The animals were submitted to a chronic variable stress (CVS) regime over a 56-day period or remained in their home cages without stress manipulation [40]. The CVS is described in Table I. 
Table I. Protocol for induction of chronic variable stress (CVS) in 56 days for the rat model

\begin{tabular}{|c|c|}
\hline Day & Stressor applied \\
\hline 1 & Cold restraint (1.5 h) \\
\hline 2 & Inclination of home cages ( $4 \mathrm{~h})$ \\
\hline 3 & Flashing light (2 h) \\
\hline 4 & Restraint (2 h) \\
\hline 5 & Isolation \\
\hline 6 & Isolation \\
\hline 7 & Isolation \\
\hline 8 & Damp bedding $(2 \mathrm{~h})$ \\
\hline 9 & Inclination of home cages ( $6 \mathrm{~h})$ \\
\hline 10 & No stressor applied \\
\hline 11 & Flashing light (2 h) \\
\hline 12 & Water deprivation (24 h) \\
\hline 13 & Restraint (3 h) \\
\hline 14 & Damp bedding ( $3 \mathrm{~h})$ \\
\hline 15 & Inclination of home cages (4 h) \\
\hline 16 & Cold restraint (2 h) \\
\hline 17 & Flashing light (3 h) \\
\hline 18 & Restraint $(2.5 \mathrm{~h})$ \\
\hline 19 & Damp bedding $(3 \mathrm{~h})$ \\
\hline 20 & Isolation \\
\hline 21 & Isolation \\
\hline 22 & Isolation \\
\hline 23 & Cold restraint (1.5 h) \\
\hline 24 & Water deprivation (24 h) \\
\hline 25 & Inclination of home cages ( $4 \mathrm{~h})$ \\
\hline 26 & Restraint (3 h) \\
\hline 27 & Flashing light (3 h) \\
\hline 28 & Restraint (1 h) \\
\hline
\end{tabular}

\begin{tabular}{|c|c|}
\hline Day & Stressor applied \\
\hline 29 & Damp bedding $(2 \mathrm{~h})$ \\
\hline 30 & No stressor applied \\
\hline 31 & Water deprivation (24 h) \\
\hline 32 & Inclination of home cages $(6 \mathrm{~h})$ \\
\hline 33 & Flashing light (2 h) \\
\hline 34 & Cold restraint (2 h) \\
\hline 35 & Isolation \\
\hline 36 & Isolation \\
\hline 37 & Isolation \\
\hline 38 & Flashing light (3 h) \\
\hline 39 & Damp bedding $(2 \mathrm{~h})$ \\
\hline 40 & Restraint (3 h) \\
\hline 41 & Cold restraint $(1.5 \mathrm{~h})$ \\
\hline 42 & Inclination of home cages ( $4 \mathrm{~h})$ \\
\hline 43 & Flashing light (2 h) \\
\hline 44 & Restraint (2 h) \\
\hline 45 & Isolation \\
\hline 46 & Isolation \\
\hline 47 & Isolation \\
\hline 48 & Damp bedding (2 h) \\
\hline 49 & Inclination of home cages (6 h) \\
\hline 50 & No stressor applied \\
\hline 51 & Flashing light (2 h) \\
\hline 52 & Water deprivation (24 h) \\
\hline 53 & Restraint (3 h) \\
\hline 54 & Damp bedding (3 h) \\
\hline 55 & Inclination of home cages ( $4 \mathrm{~h})$ \\
\hline 56 & Cold restraint (2 h) \\
\hline
\end{tabular}

\section{Tissue preparation}

The rats were deeply anesthetized and quickly decapitated. Then, the brain was exposed by an incision along the midline of the skull. A small amount of fixative was poured on the exposed brain immediately. Afterwards, the brains were removed and the left medial prefrontal cortex was dissected out $4.70-2.70 \mathrm{~mm}$ ventral and $2.20-1.40 \mathrm{~mm}$ dorsal to the Bregma [26].

\section{Estimation of total length of dendrite per neuron}

Length estimation should be done on vertical uniform random sections [1,9-11,15]. Briefly, 9-10 cyl-

inders were punched out using a trocar with $1 \mathrm{~mm}$ diameter perpendicular to the pial surface of the mPFC cortex (Fig. 1). All the cylinders of each animal were randomly rotated along their vertical axes and embedded in one paraffin block. Then, $100 \mu \mathrm{m}$ thickness slabs were obtained using a microtome. The brain samples were transferred into freshly prepared chromatin solution containing 3\% potassium dichromate and $4 \%$ or $5 \%$ paraformaldehyde in either distilled water (i.e., not buffered) or PBS solutions with $\mathrm{pH}$ of 5.8, 7.4, or 7.6. The chromatin solutions also contained both $2 \%$ glutaraldehyde and $2 \%$ chloral hydrate. All the chromatin solutions were daily changed with freshly prepared solutions. After being kept in chromatin for 48 hours, the brains 

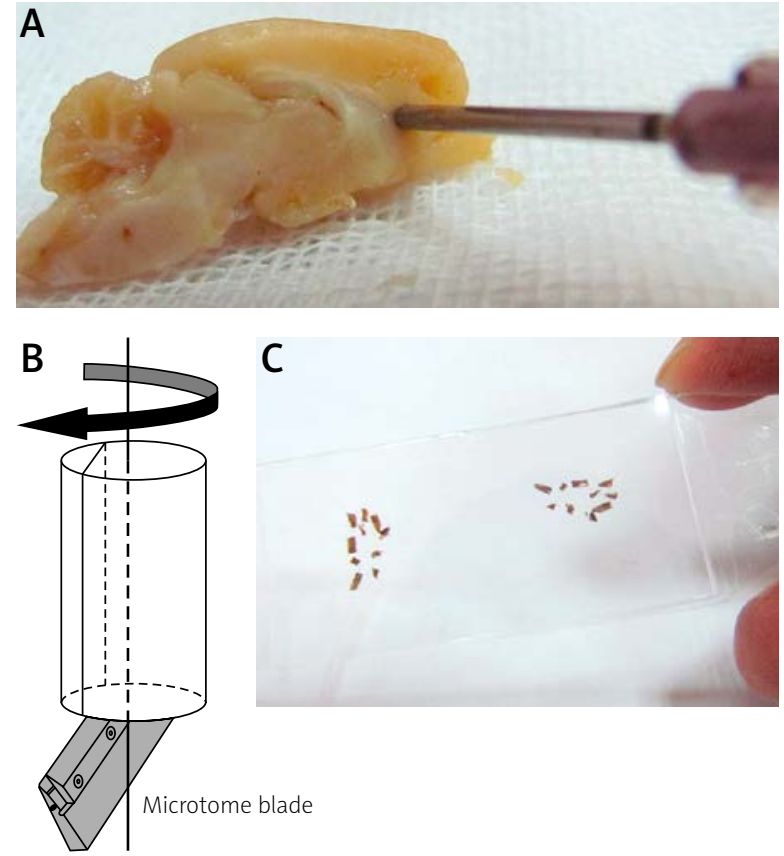

Fig. 1. Vertical uniform random sectioning. A) The vertical cylinders were punched out from the mPFC cortex vertical to its pial surface. B-C) The cylinder was randomly rotated along its vertical axis. The cylinders were sectioned using a microtome and mounted on a slide. were washed several times with a $2 \%$ silver nitrate solution in distilled water before being incubated in silver nitrate for another 48 hours for heavy metal precipitation to occur. During the whole staining process, the brains were covered with aluminum foil to avoid light exposure [7]. Mean dendritic length per neuron was calculated using the following formula [11]:

\section{$\bar{I}_{\mathrm{N}}=\frac{\text { Total dendritic length in the population }}{\text { Total number of neurons in the population }}$}

To estimate the length, a vertical section was considered. A cycloid grid and a counting frame were superimposed on the live images of the mPFC parallel to the vertical axis of the cylinder. Using a microscope (Nikon E-200) equipped with an objective lens (100x, numerical aperture of 1.4) connected to a computer, a fixed slab height of $T$ (here $100 \mu \mathrm{m}$ ) was scanned inside the section thickness (Fig. 2). To estimate the dendrite length per neuron, two quantities were measured: i) the number $\left(\mathrm{Q}^{-}\right)$of cell bodies of the neurons using the optical disector method, and ii) the total number of intersections ( $\mathrm{I}$ ) between the dendrite axes and the
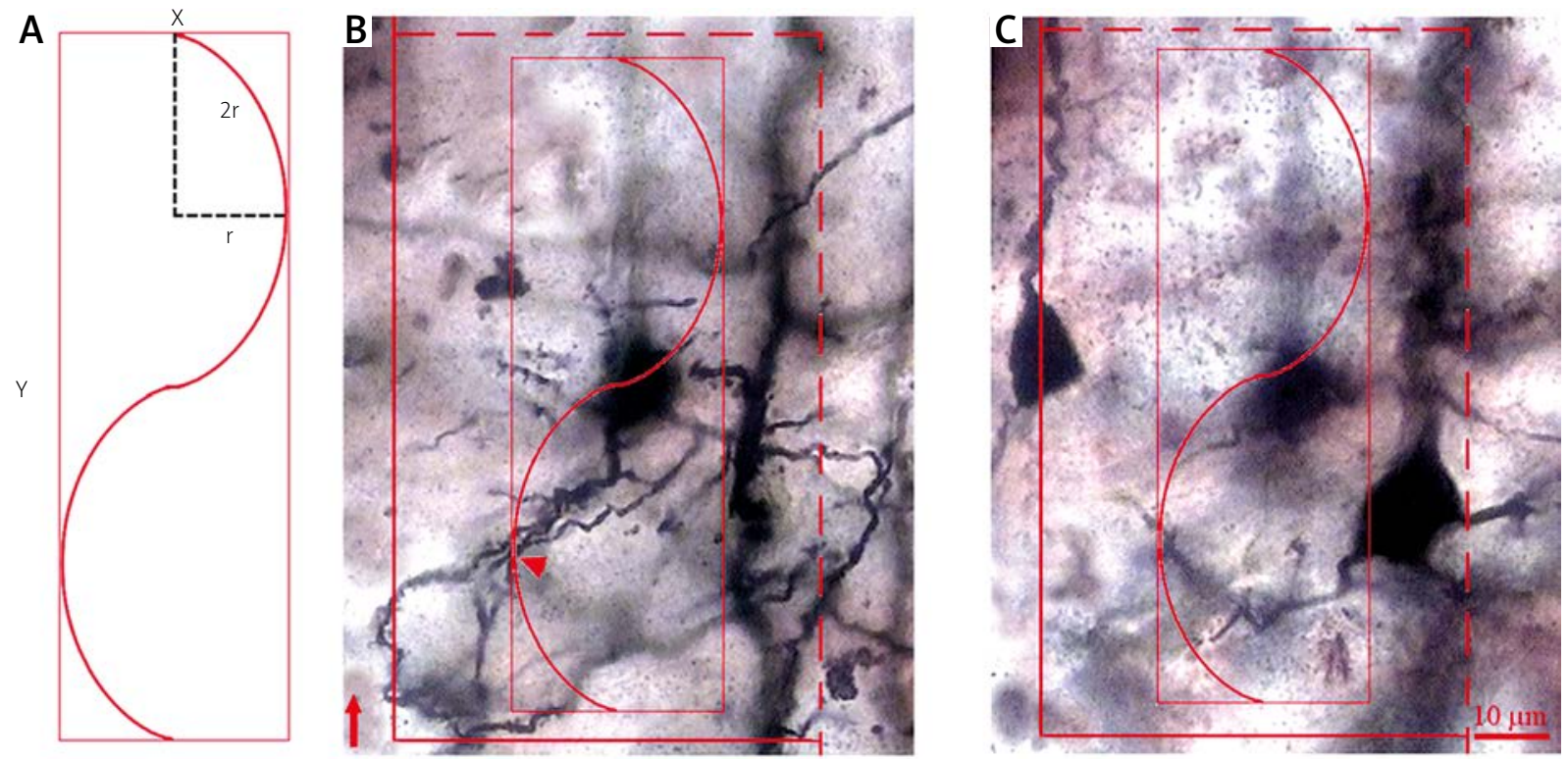

Fig. 2. Estimation of dendrite length. A) Four cycloids were located at a rectangle. The length of each cycloid was equal to twice the length of its minor axis $(r)$. The area associated with the cycloids was calculated by multiplying $X$ by $Y$ and dividing by the length of the four cycloids to achieve the area per length. B-C) When the sections were scanned, the number of cell bodies of the neurons was counted using the optical disector method and unbiased counting frame. The total number of intersections between the dendrite axes and the cycloid was counted (arrow head). The cycloid was positioned parallel to the vertical axis (arrow). 
oriented cycloid (Fig. 2) [1,9-11,15]. The following formula was used:

$\overline{\mathrm{I}}_{\mathrm{N}}=2 \cdot \frac{\mathrm{a}}{\mathrm{l}} \cdot \frac{1}{\mathrm{asf}} \cdot \mathrm{M}^{-1} \cdot \frac{\sum \mathrm{l}}{\sum \mathrm{Q}^{-}}$

Where "al" is the test area per cycloid test length, "asf" is the area associated with the cycloid grid divided by the area of the counting frame, and " $M$ " is the final magnification at $\times 4000$.

\section{Estimation of density and morphology of dendritic spines}

To estimate the density and morphology of spines, the above-mentioned dendrites were considered. Dendritic spines were identified as small protrusion that extended less than $3 \mu \mathrm{m}$ from the parent dendrite. Dendritic protrusion was classified as spines when they exhibited a characteristic enlargement at the tip, including stubby form or mushroom-type spines. The spines without enlargement were defined as thin filopodia-like protrusions [4]. Spines were counted only if they appeared continuous with the parent dendrite. Density and morphology of spines were quantified and expressed as the number of spines per neuron [4].

\section{Estimation of mean volume of neuron and nucleus}

The volume was estimated using the nucleator method. The mPFC was cut into isotropic uniform random pieces using the orientator method $[9,10]$. They were embedded in a paraffin block, sectioned (25 $\mathrm{mm}$ thickness) and stained with cresyl violet. The neurons were sampled using an optical disector $[9,10]$. For each sampled nucleolus, two horizontal directions (intercept, In) were considered from the central point within the nucleolus to the cell or nucleus borders (Fig. 3). From a series of these measurements (120-200 intercepts in each group), the mean nucleus and cell volume in the number weighted distribution was estimated using $[9,10]$ :

$\mathrm{V}_{\mathrm{N}}=\frac{4 \pi}{3} \times \overline{1_{n}^{3}}$

\section{Estimation of mean surface area of neuron}

The mean surface area of the neurons was estimated using the surfactor method. As mentioned ear- lier, to estimate the mean cell volume, the isotropic intercept emanating from the nucleus to the border of the particle was measured on the sampled cells using the disector principle (In) (Fig. 3). The acute angle $\beta(0<\beta \leq \pi / 2)$ between the intercept length and the tangent to the boundary of the cell at the point of the intersection was also measured. Finally, the surface area was measured using the following formula:

$S_{v}=4 \pi \times \bar{I}_{n}^{2} \times c(\beta), c \beta=1+\left(\frac{\pi}{2}-\beta\right) \times \cot \beta$

For the sake of simplicity, the angles were classified by the transparent protractor overlaid on the images. The protractor was a half circle which was divided into twelve $15^{\circ}$ classes (Fig. 3) [13].

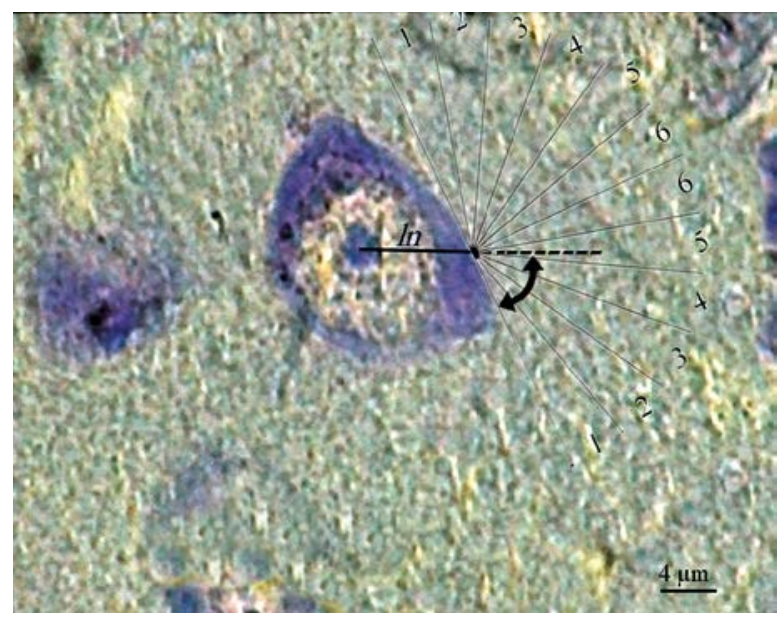

Fig. 3. Nucleator and surfactor methods. A cell is sampled using an optical disector. For each sampled nucleolus, right and left horizontal directions are considered from the central point within the nucleolus (only right is presented here). The distance (intercept, In) in both directions from the point to the boundary of the nucleus and the neuron borders is recorded and used for volume estimation. For surface area estimation, the isotropic intercept emanating from the nucleolus to the border of the particle is measured on the sampled cells (In). The acute angle " $\beta$ " (curved arrow) between the intercept length and the tangent to the boundary of the cell at the point of the intersection is shown. The angle is classified by the protractor, which was divided into twelve $15^{\circ}$ classes (here 5). 


\section{Statistical analysis}

The data were analyzed using the Kruskal-Wallis test and the Mann-Whitney $U$-test. $P \leq 0.05$ was considered as statistically significant.

\section{Results}

\section{Total length of dendrite per neuron}

The results showed that dendritic length of the mPFC was reduced by $15 \%$ in the stress + olive oil in comparison with the stress + curcumin groups $(p<$ $0.004)$. Further analysis revealed a significant decrease of $16 \%$ in the stress + distilled water in comparison with the stress + sertraline groups $(p<0.004)$ (Fig. 4).

\section{Density and morphology of spines}

The analysis of dendritic spine densities in the mPFC showed that the stress reduced the spine densities. The results showed that total spine density was reduced by $50 \%$ on average in the stress (+ olive oil or + distilled water) groups in comparison with the control group $(p<0.01)$ (Fig. 5). Analysis of spine morphology showed that density of the thin spines per neuron was reduced by $60 \%$ on average in stress (+ olive oil or + distilled water) rats in comparison to the control rats $(p<0.01)$ (Fig. 5). The density of the mushroom spines was reduced by $40 \%$ on average in stress (+ olive oil or + distilled water) rats in comparison to the control rats $(p<0.01)$ (Fig. 5). The stubby spines of the mPFC remained unchanged (Fig. 5). The data revealed that treatment of the stressed rats with curcumin and sertraline prevents spine loss.

\section{Volume and surface area of neurons}

Mean volume of the neuron and nuclei in the mPFC was decreased by $14 \%$ on average in the stress (+ distilled water or + olive oil) rats in comparison to the control rats $(p<0.01)$ (Fig. 6). Mean surface area of the neuron was decreased by $10 \%$ on average in the stress (+ distilled water or + olive oil) rats in comparison to the control rats $(p<0.01)$ (Fig. 6).

The data revealed that treatment of the stressed rats with curcumin or sertraline prevents reduction of the volume and surface area of the neurons.

\section{Discussion}

The present study investigated the morphological changes of the dendrites and neurons in MPFC as a result of chronic variable stress using stereological methods. The results showed that chronic stress changes the morphology of dendrites and neurons in the mPFC. Moreover, this adverse stress effect was shown to be prevented by treatment with curcumin and sertraline.

Stress-induced remodeling in the MPFC has been suggested to have distinct functional consequences [18]. Therefore, evaluation of the dendritic morphology is a valuable parameter. The reduction in the dendritic length per neuron, density and morphology of spine is in accordance with the studies conducted by Cook and Wellman [5], Izquierdo et al. [12], and Shansky et al. $[38,39]$ showing stress to induce significant changes in the morphology of the pyramidal neurons and decrease in length of dendrites or retraction of

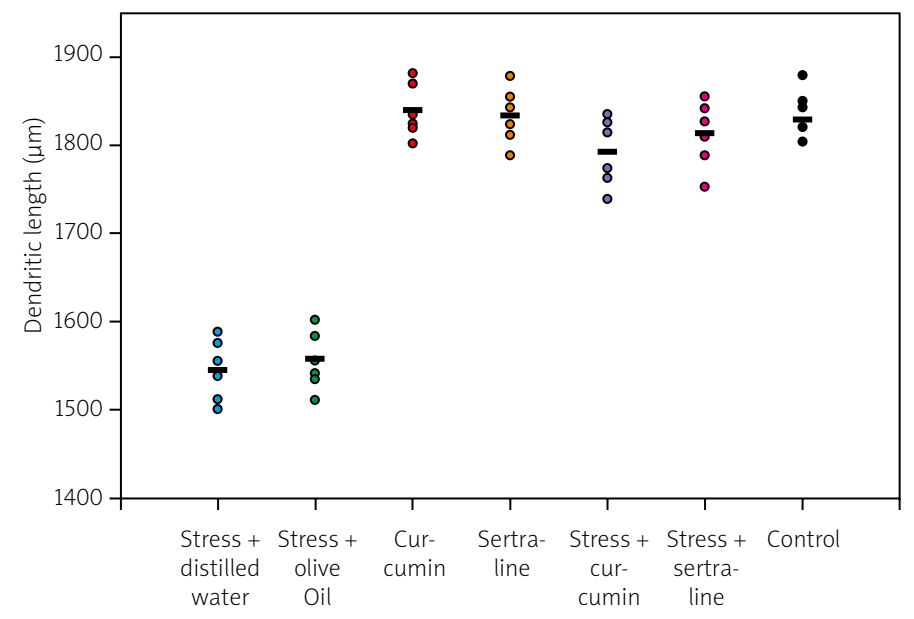

Fig. 4. Length of dendrites. Dot plot showing the total length of the dendrites per neuron in different groups including control, distilled water, olive oil, curcumin, sertraline with or without stress treatment. 

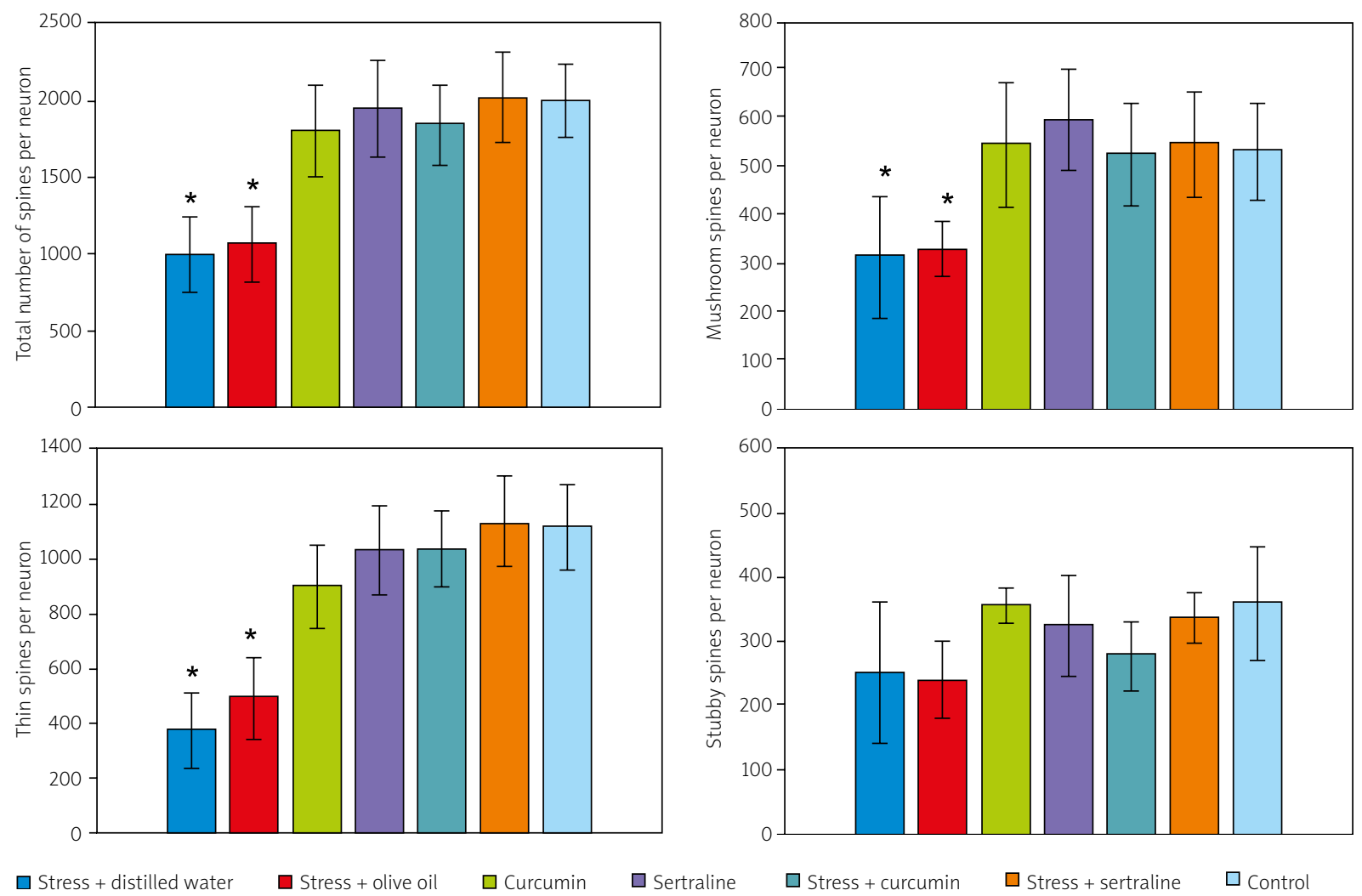

Fig. 5. Density of spines. The total density and the density of different spine types (thin, mushroom and stubby) per neuron of the mPFC in the different groups are shown. The significant difference between stress (+ olive oil and + distilled water) with the other groups is indicated. ${ }^{*} p<0.01$.

the apical dendritic arbor in the pyramidal cells in the PFC. These changes in the dendritic length, density and morphology of the spine have been shown to be accompanied by impairments in the cognitive functions selectively mediated by the MPFC [18].

In general, the length estimation method mostly relies on tracing images of the dendrites. However, the technique presented here is a design-based stereological method and does not require tracing, which is a time-consuming procedure. In addition, tracing might be vulnerable to mistake when the branches cannot be traced on the obscure sides of the dendrites.

Our study revealed that chronic exposure to stress reduced the spine density in the mPFC and also reduced the thin and mushroom spines in MPFC, whereas stubby spines in the MPFC remained unchanged. However, it is hypothesized that thin and mushroom spines represent learning spines and memory spines, respectively. It is the result of their constant changing in response to neuronal activ- ity (e.g., chronic stress) [36]. This is in accordance with our previous study showing that stress impairs learning and memory [23].

The present study also indicated that chronic exposure to stress reduced the volume of neurons, neuron nuclei and surface area of neurons in the mPFC. Furthermore, clinical studies showed that structural brain modifications in depressed patients are similar to those found in animal models of chronic variable stress (such as decreased neuronal soma size and neuronal density) in the mPFC. The findings of this study are thus in line with previous reports of reduced neuronal soma size in the prefrontal cortex. Reduced neuronal soma size is also described in major depressive disorder [29,32]. The surface area of the neuron is important in their functions and synaptic activities. However, this parameter has received less attention in histopathological research.

The chronic stress-induced factors that lead to these changes are not fully understood. Nevertheless, previous studies have shown that repeat- 

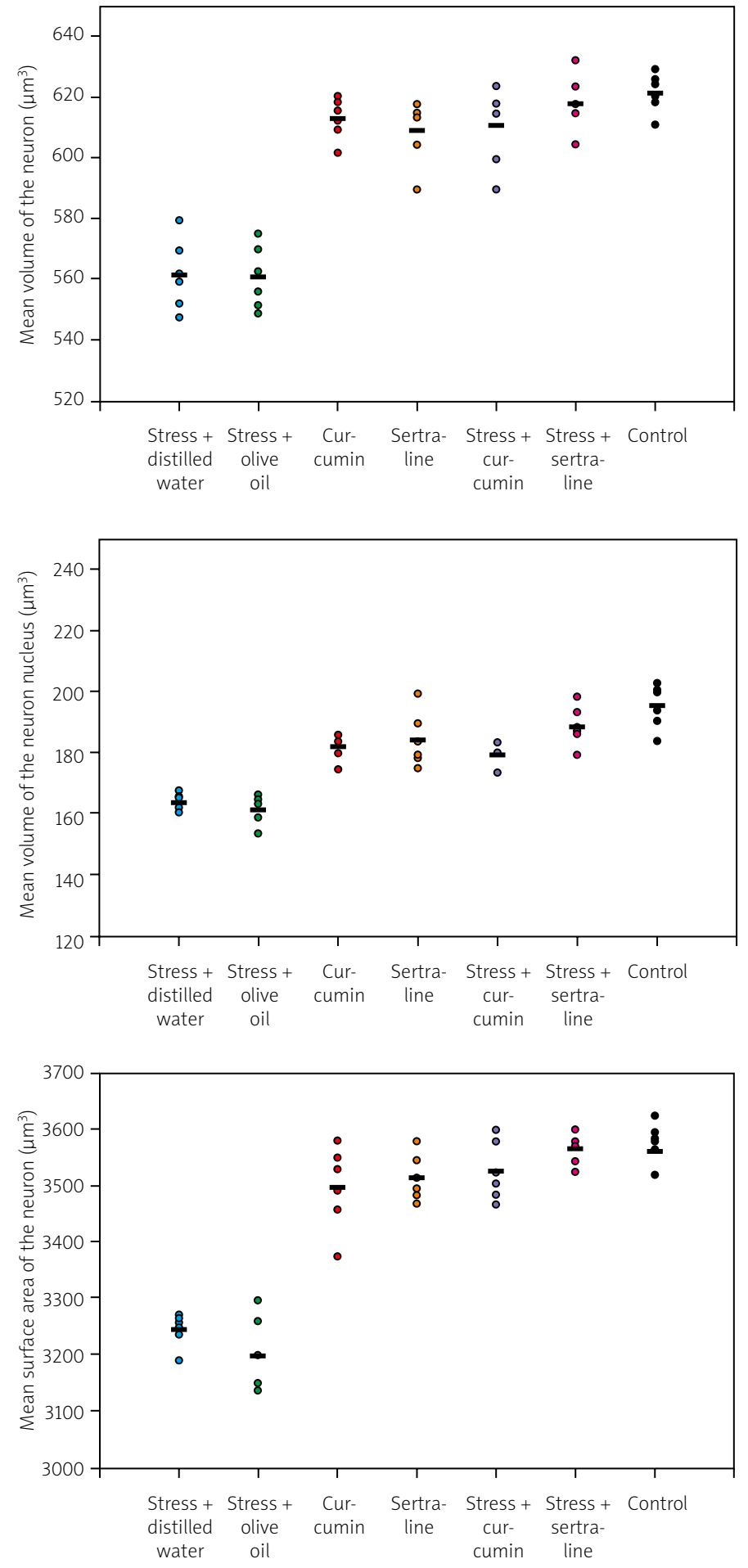

Fig. 6. Volume and surface area. Dot plot showing mean volume of the neuron, mean volume of the neuron nuclei and mean surface area of the neuron of the mPFC in different groups. 
ed stress reduced dopamine [22,27], noradrenalin [14], and serotonin in the mPFC [20]. Experimental findings also emphasize that serotonin changes spine density and dendrite length in the MPFC by acting on 5-HT receptors [21]. The MPFC seems to be severely sensitive and reacts faster to stressful events [41]. Glucocorticoid receptors are present in the mPFC of rats. Also, catecholamines and glucocorticoids are the key mediators of the stress response and release upon the nervous system [8,34]. The medial prefrontal cortex plays a key role in controlling the hypothalamic-pituitary-adrenal axis and regulates the stress response of other structures [8]. The present study also demonstrated that sertraline and curcumin had protective effects on the morphological changes of dendrites and neurons of the mPFC destroyed by stress. Selective serotonin reuptake inhibitors are the most widely prescribed antidepressants today and exert their antidepressant-like effects by inhibiting the neuronal reuptake of serotonin and increasing the synaptic concentrations of serotonin. Sertraline treatment also reduced acetylcholinesterase enzyme levels in all regions of the brain [43]. Previous studies showed that fluoxetine (a selective serotonin reuptake inhibitor) could cause an increase in the total dendritic length in CA1 but not in the dentate gyrus [25]. Our finding is also in accordance with these reports. The effect of curcumin was also evaluated and compared with sertraline in the present investigation. This compound showed the same protective effects as sertraline on dendrite length after stress induction. Although the mechanism of the antidepressant effect of curcumin is not completely understood [16], previous studies have reported that antidepressant effects may be obtained by several mechanisms, such as inhibition of serotonin uptake [6,33]. In addition, curcumin is an antidepressant which is well proven in inhibiting the monoamine oxidase enzyme and modulating the release of serotonin and dopamine [16]. However, evidence has shown that curcumin administration also increased hippocampal neurogenesis in chronically stressed rats by modulation of the hypothalamic-pituitary-adrenal axis and regulation of 5-HT1A receptors as well as the brain-derived neurotrophic factor in the hippocampus [42]. This idea is supported by the finding that antidepressants can promote neurogenesis [42]. As it appears, different factors might be effective in curcumin's mechanism of action. However, to our knowledge, no studies have been conducted on the effects of curcumin on the dendritic and neuron morphology after stress. In conclusion, the present findings demonstrate that curcumin and sertraline could protect against the adverse effects of stress on dendritic length, density, morphology of the spine, volume of neurons, volume of neuron nuclei and surface area of neurons of the mPFC.

\section{Acknowledgements}

The work was performed at the Histomorphometry and Stereology Research Centre, Shiraz University of Medical Sciences, Shiraz, Iran. This study is part of a thesis written by Mohammad-Amin Abdollahifar, $\mathrm{PhD}$ student of Anatomy. The work was financially supported by the Research Vice-Chancellor of Shiraz University of Medical Sciences (Grant No. 91-6124). The authors would like to thank RouzDarou Pharmaceutical Company for their kind provision of sertraline. They are also grateful to the Research Improvement Center of Shiraz University of Medical Sciences and Ms. A. Keivanshekouh for improving the use of English in the manuscript.

\section{Disclosure}

Authors report no conflict of interest.

\section{References}

1. Baddeley AJ, Gundersen HJ, Cruz-Orive LM. Estimation of surface area from vertical sections. J Microsc 1986; 142: 259-276.

2. Boda B, Alberi S, Nikonenko I, Node-Langlois R, Jourdain P, Moosmayer M, Parisi-Jourdain L, Muller D. The mental etardation protein PAK3 contributes to synapse formation and plasticity in hippocampus. J Neurosci 2004; 24: 10816-10825.

3. Brouet I, Ohshima H. Curcumin, an anti-tumour promoter and anti-inflammatory agent, inhibits induction of nitric oxide synthase in activated macrophages. Biochem Biophys Res Commun 1995; 206: 533-540.

4. Chapleau CA, Carlo ME, Larimore JL, Pozzo-Miller L. The actions of BDNF on dendritic spine density and morphology in organotypic slice cultures depend on the presence of serum in culture media. J Neurosci Methods 2008; 169: 182-190.

5. Cook SC, Wellman CL. Chronic stress alters dendritic morphology in rat medial prefrontal cortex. J Neurobiol 2004; 60: 236248.

6. Cryan JF, Leonard BE. 5-HT1A and beyond: the role of serotonin and its receptors in depression and the antidepressant response. Hum Psychopharmacol 2000; 15: 113-135.

7. de Ruiter JP, Uylings HB. Morphometric and dendritic analysis of fascia dentata granule cells in human aging and senile dementia. Brain Res 1987; 402: 217-229. 
8. Diorio D, Viau V, Meaney MJ. The role of the medial prefrontal cortex (cingulate gyrus) in the regulation of hypothalamic-pituitary-adrenal responses to stress. J Neurosci 1993; 13: 3839-3847.

9. Gundersen HJ, Bagger P, Bendtsen TF, Evans SM, Korbo L, Marcussen N, Møller A, Nielsen K, Nyengaard JR, Pakkenberg B, et al. The new stereological tools: disector, fractionator, nucleator and point sampled intercepts and their use in pathological research and diagnosis. APMIS 1988; 96: 857-881.

10. Gundersen HJ, Bendtsen TF, Korbo L, Marcussen N, Møller A, Nielsen K, Nyengaard JR, Pakkenberg B, Sørensen FB, Vesterby A et al. Some new, simple and efficient stereological methods and their use in pathological research and diagnosis. APMIS 1988; 96: 379-394.

11. Howard CV, Cruz-Orive LM, Yaegashi H. Estimating neuron dendritic length in 3D from total vertical projections and from vertical slices. Acta Neurol Scand Suppl 1992; 137: 14-19.

12. Izquierdo A, Wellman CL, Holmes A. Brief uncontrollable stress causes dendritic retraction in infralimbic cortex and resistance to fear extinction in mice. J Neurosci 2006; 26: 5733-5738.

13. Karlsson LM, Cruz-Orive LM. Estimation of mean particle size from single sections. J Micros 1997; 186: 121-132.

14. Kitayama I, Kayahara T, Nakano K, Murase S, Otani M, Nomura J. Long-term stress degenerates, but imipramine regenerates, noradrenergic axons in the rat cerebral cortex. Biol Psychiatry 1997; 42: 687-696.

15. Kristiansen SL, Nyengaard JR. Digital stereology in neuropathology. APMIS 2012; 120: 327-340

16. Kulkarni SK, Bhutani MK, Bishnoi M. Antidepressant activity of curcumin: involvement of serotonin and dopamine system. Psychopharmacology (Berl) 2008; 201: 435-442.

17. Kulkarni S, Dhir A, Akula KK. Potentials of curcumin as an antidepressant. ScientificWorldJournal 2009; 9: 1233-1241.

18. Liston C, Miller MM, Goldwater DS, Radley JJ, Rocher AB, Hof PR, Morrison JH, McEwen BS. Stress-induced alterations in prefrontal cortical dendritic morphology predict selective impairments in perceptual attentional set-shifting. J Neurosci 2006; 26 7870-7874

19. Lorenzetti V, Allen NB, Fornito A, Yücel M. Structural brain abnormalities in major depressive disorder: a selective review of recent MRI studies. J Affect Disord 2009; 117: 1-17.

20. Mangiavacchi S, Masi F, Scheggi S, Leggio B, De Montis MG, Gambarana C. Long-term behavioral and neurochemical effects of chronic stress exposure in rats. J Neurochem 2001; 79: 11131121.

21. Miner LA, Backstrom JR, Sanders-Bush E, Sesack SR. Ultrastructural localization of serotonin2A receptors in the middle layers of the rat prelimbic prefrontal cortex. Neuroscience 2003; 116 : 107-117.

22. Mizoguchi K, Yuzurihara M, Ishige A, Sasaki H, Chui DH, Tabira T. Chronic stress induces impairment of spatial working memory because of prefrontal dopaminergic dysfunction. J Neurosci 2000; 20: 1568-1574

23. Noorafshan A, Abdollahifar MA, Karbalay-Doust S, Asadi-Golshan R, Rashidiani-Rashidabadi A. Protective effects of curcumin and sertralineon the learning and memory impairment, anxiety, and anhedoniain chronic stressed rats. Exp Neurobiol 2013; 22: 96-106.
24. Noorafshan A, Asadi-Golshan R, Karbalay-Doust S, Abdollahifar MA, Rashidiani-Rashidabadi A. Curcumin, the main part of turmeric, prevents learning and memory changes induced by sodium metabisulfite, a preservative agent, in rats. Exp Neurobiol 2013; 22: 23-30.

25. Norrholm SD, Ouimet CC. Chronic fluoxetine administration to juvenile rats prevents age-associated dendritic spine proliferation in hippocampus. Brain Res 2000; 883: 205-215.

26. Paxinos G, Watson C. The rat brain in stereotaxic coordinates. Academic Press, San Diego 2007.

27. Pascucci T, Ventura R, Latagliata EC, Cabib S, Puglisi-Allegra S. The medial prefrontal cortex determines the accumbens dopamine response to stress through the opposing influences of norepinephrine and dopamine. Cereb Cortex 2007; 17: 2796-2804.

28. Perez-Cruz C, Simon M, Flügge G, Fuchs E, Czéh B. Diurnal rhythm and stress regulate dendritic architecture and spine density of pyramidal neurons in the rat infralimbic cortex. Behav Brain Res 2009; 205: 406-413.

29. Pierri JN, Volk CL, Auh S, Sampson A, Lewis DA. Decreased somal size of deep layer 3 pyramidal neurons in the prefrontal cortex of subjects with schizophrenia. Arch Gen Psychiatry 2001; 58: 466-473.

30. Radley JJ, Rocher AB, Miller M, Janssen WG, Liston C, Hof PR, McEwen BS, Morrison JH. Repeated stress induces dendritic spine loss in the rat medial prefrontal cortex. Cereb Cortex 2006; 16: 313-320.

31. Radley JJ, Sisti HM, Hao J, Rocher AB, McCall T, Hof PR, McEwen BS, Morrison JH. Chronic behavioral stress induces apical dendritic reorganization in pyramidal neurons of the medial prefrontal cortex. Neuroscience 2004; 125: 1-6.

32. Rajkowska G, Miguel-Hidalgo JJ, Wei J, Dilley G, Pittman SD, Meltzer HY, Overholser JC, Roth BL, Stockmeier CA. Morphometric evidence for neuronal and glial prefrontal cell pathology in major depression. Biol Psychiatry 1999; 45: 1085-1098.

33. Rush AJ, Trivedi MH, Wisniewski SR, Stewart JW, Nierenberg AA, Thase ME, Ritz L, Biggs MM, Warden D, Luther JF, Shores-Wilson K, Niederehe G, Fava M; STAR*D Study Team. Bupropion-SR, sertraline, or venlafaxine-XR after failure of SSRIs for depression. N Engl J Med 2006; 354: 1231-1242.

34. Sánchez MM, Young LJ, Plotsky PM, Insel TR. Distribution of corticosteroid receptors in the rhesus brain: relative absence of glucocorticoid receptors in the hippocampal formation. J Neurosci 2000; 20: 4657-4668.

35. Sanmukhani J, Anovadiya A, Tripathi CB. Evaluation of antidepressant like activity of curcumin and its combination with fluoxetine and imipramine: an acute and chronic study. Acta Pol Pharm 2011; 68: 769-775.

36. Segal I, Korkotian I, Murphy DD. Dendritic spine formation and pruning: common cellular mechanisms? Trends Neurosci 2000; 23: 53-57.

37. Selye $H$. The evolution of the stress concept. Am Sci 1973; 61: 692-699.

38. Shansky RM, Hamo C, Hof PR, McEwen BS, Morrison JH. Stress-induced dendritic remodeling in the prefrontal cortex is circuit specific. Cereb Cortex 2009; 19: 2479-2484.

39. Shansky RM, Morrison JH. Stress-induced dendritic remodeling in the medial prefrontal cortex: effects of circuit, hormones and rest. Brain Res 2009; 1293: 108-113. 
40. Tagliari B, Noschang CG, Ferreira AG, Ferrari OA, Feksa LR, Wannmacher CM, Dalmaz C, Wyse AT. Chronic variable stress impairs energy metabolism in prefrontal cortex and hippocampus of rats: prevention by chronic antioxidant treatment. Metab Brain Dis 2010; 25: 169-176.

41. Vertes RP. Differential projections of the infralimbic and prelimbic cortex in the rat. Synapse 2004; 51: 32-58.

42. Xu Y, Ku B, Cui L, Li X, Barish PA, Foster TC, Ogle WO. Curcumin reverses impaired hippocampal neurogenesis and increases serotonin receptor $1 \mathrm{~A}$ mRNA and brain-derived neurotrophic factor expression in chronically stressed rats. Brain Res 2007; 1162: 9-18.

43. Yildirim E, Erol K, Ulupinar E. Effects of sertraline on behavioral alterations caused by environmental enrichment and social isolation. Pharmacol Biochem Behav 2012; 101: 278-287. 